\title{
Unusual Bowel Perforation Following Dilatation and Curettage in a Case of Endometrial Cancer
}

\author{
K. Samita Bhat ${ }^{1}$ (D) Vijay K. Ahuja ${ }^{1}$ - S. P. Somashekhar ${ }^{1}$ - Susmita H. Rakshit ${ }^{2}$
}

Received: 6 March 2018 / Accepted: 3 April 2018/Published online: 13 April 2018

(C) The Author(s) 2018

\begin{abstract}
We present an unusual case of iatrogenic small bowel perforation in a woman with endometrial cancer. A 57-year-old postmenopausal woman with past history of total colostomy with loop ileostomy for ulcerative colitis, was referred to our department for evaluation and further management of suspected endometrial cancer following dilatation and curettage (D\&C) elsewhere. The histopathology showed intestinal element which was attributed to metaplasia; however, no malignancy was identified in the biopsy specimen. Imaging carried out elsewhere after D\&C showed thickened endometrium with suspicious small bowel infiltration. The patient was completely asymptomatic with no signs of bowel injury or peritonitis. After a complete evaluation, a class 1 extrafascial hysterectomy and bilateral salpingo-oophorectomy with segmental ileal resection and anastomosis and frozen section were performed. Intraoperatively, small bowel loop was found adherent to the posterior uterine wall extending up to the cervix with no obvious sign of any uterine or bowel perforation. The final histopathology revealed endometrioid adenocarcinoma grade 1, stage IA, with tumor located at the fundus along with cervical isthmic perforation on the posterolateral wall with full thickness ileum perforation. Uterine perforation at $\mathrm{D} \& \mathrm{C}$ is a known complication, but a silent bowel injury presenting with intestinal tissue in endometrial biopsy is very unusual. A high degree of suspicion is required to detect such complications at D\&C especially in postmenopausal women with past history of abdominal surgery.
\end{abstract}

Keywords Dilatation and curettage $(\mathrm{D} \& \mathrm{C}) \cdot$ Bowel injury $\cdot$ Intestinal element in histopathology $\cdot$ Endometrial cancer

\section{Introduction}

Endometrial biopsy is a routine and safe surgical procedure in gynecology. Complications are rare but can be fatal at times [1]. Uterine perforation can occur during D\&C by sounding the uterus or during the cervical dilatation [2]. Perforation can also occur during curettage especially in case of previous uterine scar or in the presence of endometrial carcinoma causing weakness in the uterine wall making it amenable to perforation. During D\&C for non-obstetric reasons, perforation has been reported in

\section{K. Samita Bhat}

samitasundeep@gmail.com

1 Manipal Comprehensive Cancer Centre, Department of Surgical Oncology, Manipal Hospitals, \#98, HAL Airport Road, Bangalore 560017, India

2 Department of Pathology, Manipal Hospitals, HAL Airport Road, Bangalore, India approximately $0.3 \%$ in premenopausal woman and $2.6 \%$ in the postmenopausal woman [3]. Out of all complications, full thickness bowel perforation after D\&C is a unique and serious complication, but asymptomatic cases are very rare $[4,5]$. Diagnosis is by strong a clinical suspicion, and the need for immediate surgical intervention is based on clinical signs and symptoms of peritonitis and imaging [6]. We report a unique case of silent iatrogenic ileal perforation in a case of postmenopausal woman with endometrial carcinoma.

\section{Case Report}

A 57-year-old postmenopausal woman, P1L1, with previous spontaneous vaginal delivery presented with postmenopausal bleeding at a city hospital. She had a past history of total colectomy and permanent ileostomy 15 years back for ulcerative colitis. An ultrasound 
abdomen was done which showed thickened endometrium $(12 \mathrm{~mm})$ with no other significant findings. She was then planned for hysteroscopy and D\&C at the same hospital. Intraoperatively, hysteroscopy was abandoned due to cervical stenosis, but D\&C with polypectomy was done, and she got discharged in a stable condition on the same day. HPE reported as bits of small intestinal tissue in full thickness with mucosa and some endocervical but no endometrial tissue (Fig. 1). Intestinal element was attributed to metaplasia.

Pelvic scan (TVS) on 10th post-D\&C day shows thick $(2.4 \mathrm{~cm})$ echogenic highly vascular endometrium, irregular junctional zone with myometrial invasion up to the serosa suggestive of endometrial carcinoma.

CECT and MRI performed elsewhere reported a normalsized uterus with endometrial thickness of $2.8 \mathrm{~cm}$. A breach in the junctional zone and myometrium infiltrated posterosuperiorly on the right side with minimal extension of thickened endometrium beyond the uterine serosa was seen to involve the wall of adjacent ileal loop with intact cervix, raised the possibility of intestinal infiltration of endometrial carcinoma (Fig. 2).

Patient presented to us 30 days post-D\&C with all reports of investigations. She was completely asymptomatic on examination. Performance score (ECOG) was zero, vitals were stable, and abdomen was soft, non-tender with no sign of peritonitis. After reviewing the slides, patient was planned for staging laparotomy and frozen section, in view of radiological suspicion of endometrial carcinoma. On laparotomy, dense inter-loop adhesions between small bowel and to anterior abdominal wall were seen. An ileal segment was densely adherent to the posterior wall of uterus extending right from fundus to the cervix. Rest of the abdomen and ileostomy site on right side appeared healthy. Ileal loop was dissected off the uterine wall by sharp dissection except posteriorly at the level of cervix. Class 1 Hysterectomy and BSO with indocyanine (ICG)-based sentinel lymph node biopsy

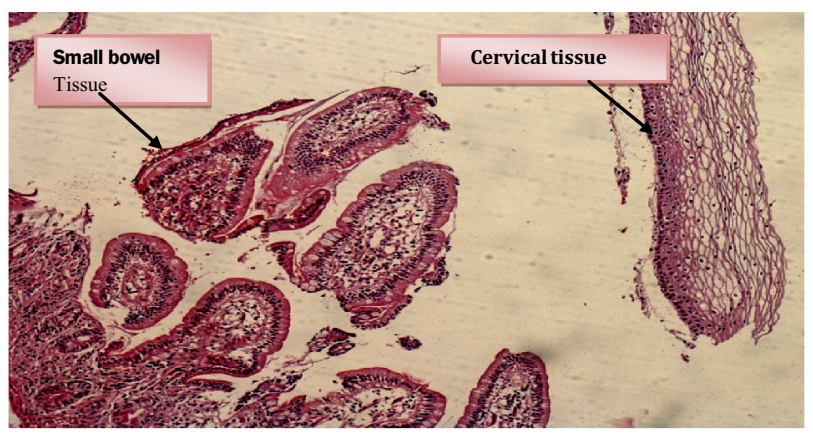

Fig. $1 \mathrm{D} \& \mathrm{C}$ specimen showing small bowel villous tissue lying beside the squamous component of cervical tissue

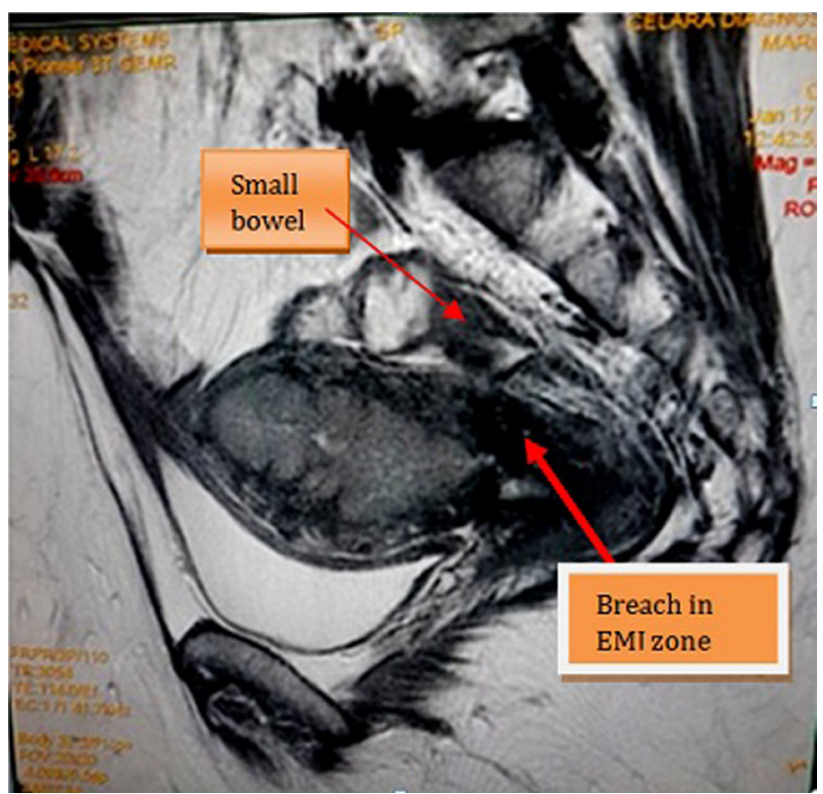

Fig. 2 MRI pelvis sagittal view

(SLNB) were performed. Segmental ileal resection and anastomosis was performed in view of dense adhesions.

Frozen section reported as endometrioid adenocarcinoma G2, with $<50 \%$ myometrial invasion and tumor size of $5 \times 3.5 \mathrm{~cm}$ with reactive nodes. In view of negative sentinel nodes, further staging lymphadenectomy was abandoned. The final histopathology showed the same findings. Sections from the adherent ileal loop showed numerous congested blood vessels in the subepithelium and no evidence of dysplasia/malignancy at the perforation site. Sections from the perforated area on in the cervix revealed a sinus tract in the cervical epithelium and intense granulation tissue. No sign of any dysplasia or malignancy was found at the perforation site (Fig. 3).

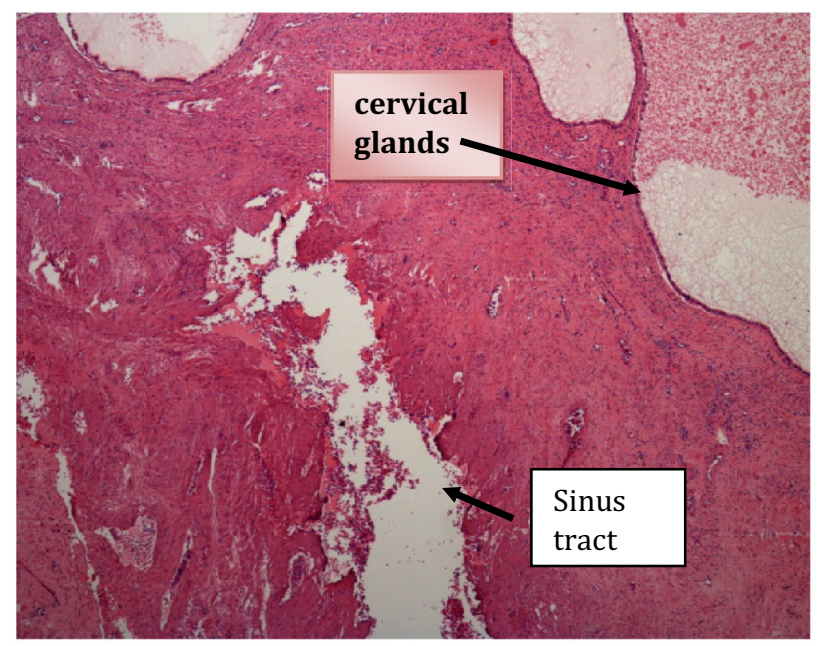

Fig. 3 Final HPE reported sinus tract post-D\&C in cervical wall 


\section{Discussion}

Uterine perforation is a potential complication of $\mathrm{D} \& \mathrm{C}$ and may be associated with injury to viscera (bladder, bowel) [7]. Factors that make access to the endometrial cavity difficult, e.g., cervical stenosis, or that alter the strength of the myometrial wall, e.g., pregnancy, lactation, and menopause, are the risk factors for uterine or bowel perforation [8]. Hafler et al. retrospectively analyzed the incidence of complications after D\&C in non-obstetric uterus in 5329 cases and found that site of uterine perforation was fundus in 47 cases and cervical perforation in three cases and concluded that a retroverted uterus, postmenopausal status, and nulliparity are independent risk factors for intraoperative complications [9].

In our case, the presence of intestinal tissue in D\&C specimen raised the following possibilities: (1) In view of imaging showing suspected bowel infiltration, there was a possibility of endometrial cancer perforating the adjacent bowel. However, the biopsy was negative for malignancy. (2) Previous surgery was for ulcerative colitis causing ileouterine fistula, but there was an absence of intestinal contents draining through the cervix or collecting in the uterus. (3) Uterine perforation was performed during $\mathrm{D} \& \mathrm{C}$, but patient was completely asymptomatic for the last 20 days starting from the date of D\&C till she approached us. Intraoperatively, also there were no definite signs of perforation. Hence, none of these could be confirmed preoperatively, and the final diagnosis of bowel perforation and endometrial cancer was made only by histological evaluation of the surgical specimen. That clearly indicates that the perforation was iatrogenic and most probably due to postmenopausal stenosed internal cervical os and distorted cervical canal.

The learning points in this case are as follows. We should always have a strong suspicion of cervical stenosis in postmenopausal women. In this case, as hysteroscopy was not possible, an option would have been to do a pipelle biopsy. Another method described is to give prostaglandin analog (PGE1) at least $2 \mathrm{~h}$ prior to hysteroscopy and D\&C for cervical priming and dilatation to avoid perforation [10]. Secondly, bowel perforation should be suspected when yellow fleshy material is curetted along with the endometrium and when patient complains of sudden pain and dragging sensation which is often ignored thinking that it is procedure-related pain. In our case though the patient was asymptomatic, but because of a previous history of colectomy with along with suspicion of endometrial carcinoma, a laparotomy was done.

\section{Conclusion}

In a postmenopausal woman with previous bowel or uterine surgery, extra caution should be exercised during dilatation and curettage. The endometrial curetting or any polyp removed should be examined carefully for unusual appearance before sending the specimen for histopathology. Management of postmenopausal uterine perforation would depend upon symptoms, signs, and findings on imaging. However, in rare instances like this case, where intestinal luminal tissue is seen in endometrial curettage sample, possibility of bowel injury should be considered rather than attributing it to metaplasia.

\section{Compliance with Ethical Standards}

Conflict of interest Authors declare that they have no conflict of interest.

Open Access This article is distributed under the terms of the Creative Commons Attribution 4.0 International License (http://creative commons.org/licenses/by/4.0/), which permits unrestricted use, distribution, and reproduction in any medium, provided you give appropriate credit to the original author(s) and the source, provide a link to the Creative Commons license, and indicate if changes were made.

\section{References}

1. Abulafia O, Shah T, Salame G, Economos K, Serur E, Zinn H, Sokolovkski M, Sherer DM. Sonographic and magnetic resonance imaging findings of pelvic abscess following uterine perforation sustained during office endometrial sampling. J Clin Ultrasound. 2011;39(5):283-6.

2. McELIN TW, Bird CC, Reeves BD, Scott RC. Diagnostic dilatation and curettage: a 20-year survey. Obstet Gynecol. 1969;33(6):807-12.

3. Dunner PS, Thomas MA, Ferreras M, Jerome M. Intrauterine incarcerated bowel following uterine perforation during an abortion: a case report. Am J Obstet Gynecol. 1983;147(8):969-70.

4. Vecchio R, Marchese S, Leanza V, Leanza A, Intagliata E. Totally laparoscopic repair of an ileal and uterine iatrogenic perforation secondary to endometrial curettage. Int Surg. 2015;100(2):244-8.

5. Coughlin LM, Sparks DA, Chase DM, Smith J. Incarcerated small bowel associated with elective abortion uterine perforation. J Emerg Med. 2013;44(3):e303-6.

6. Zissin R, Osadchy A, Gayer G. Abdominal CT findings in small bowel perforation. Br J Radiol. 2009;82(974):162-71.

7. Gentile GP, Siegler AM. Inadvertent intestinal biopsy during laparoscopy and hysteroscopy: a report of two cases. Fertil Steril. 1981;36(3):402-4.

8. Shulman SG, Bell CL, Hampf FE. Uterine perforation and small bowel incarceration: sonographic and surgical findings. Emerg Radiol. 2006;13(1):43-5.

9. Hefler L, Lemach A, Seebacher V, Polterauer S, Tempfer C, Reinthaller A. The intraoperative complication rate of nonobstetric dilation and curettage. Obstet Gynecol. 2009;113(6):1268-71.

10. Zhuo Z, Yu H, Jiang X. A systematic review and meta-analysis of randomized controlled trials on the effectiveness of cervical ripening with misoprostol administration before hysteroscopy. Int J Gynecol Obstet. 2016;132(3):272-7. 